\title{
Comprehensive Analysis of Prognostic Value and Immune Infiltration of MMP12 in Esophageal Squamous Cell Carcinoma
}

\author{
Jing-tao Mao, ${ }^{1}$ Qiang Lu, ${ }^{2}$ Peng-yu Jing, ${ }^{2}$ Zi-liang Li, ${ }^{1}$ Xiao-qi Yang, ${ }^{1}$ Ji-peng Zhang, \\ and Zhe $\mathrm{Li}^{3}{ }^{3}$ \\ ${ }^{1}$ Department of Thoracic Surgery, Shaanxi Provincial Sengong Hospital, Xi'an, Shaanxi 710300, China \\ ${ }^{2}$ Department of Thoracic Surgery, Tangdu Hospital, The Air Force Military Medical University, Xi'an, Shaanxi 710038, China \\ ${ }^{3}$ Department of Thoracic Surgery, The Ninth Hospital of Xi' an, Xi'an, Shaanxi 710054, China
}

Correspondence should be addressed to Zhe Li; lizhe197906@163.com

Received 16 January 2022; Revised 6 February 2022; Accepted 9 February 2022; Published 27 February 2022

Academic Editor: Fu Wang

Copyright ( 2022 Jing-tao Mao et al. This is an open access article distributed under the Creative Commons Attribution License, which permits unrestricted use, distribution, and reproduction in any medium, provided the original work is properly cited.

Esophageal squamous cell carcinoma (ESCC) is a typical neoplastic disease and a frequent cause of death in China. The prognosis of most ESCC patients is still poor. Previous studies demonstrated that MMP12 is involved in tumor metastasis. However, its clinical significance and association with cancer immunity remained largely unclear. In this study, we first analyzed the expressing pattern of MMPs in ESCC from TCGA datasets and found that several MMPs expression was distinctly increased in ESCC. However, only MMP12 expression was associated with five-year survival of ESCC patients. Then, we focused on MMP12 and found its high expression was positively related to advanced clinical stages of ESCC specimens. KEGG assays revealed MMP12 may influence the activity of several tumor-related pathways, such as the Toll-like receptor signaling pathway, TNF signaling pathway, and IL-17 signaling pathway. Then, we sought to determine whether MMP12 expressions were related to immune cell infiltration in ESCC. We observed that increased MMP12 levels were positively associated with the infiltration levels of mast cells activated and macrophages M0. However, eosinophils, B cells naïve, and mast cells resting exhibited an opposite result. Finally, we showed that knockdown of MMP12 suppressed the proliferation of ESCC cells. Overall, our findings proved that high expression of MMP12 may be a novel and valuable prognostic factor in ESCC.

\section{Introduction}

Esophageal squamous cell carcinoma (ESCC) remains the most prevailing histological subtype of esophageal cancer in developing nations or regions, such as China and Iran [1]. Among many types of clinical features associated with ESCC progression, distant metastases remain the critical element for unfavorable survivals [2]. Although the significant progresses have been achieved in the effective treatments of ESCC by the use of chemoradiotherapy and surgery in recent years, the 5-year survival rate remains $<40 \%[3,4]$. Consequently, it is urgent to comprehend the genetic and molecular mechanism of ESCC to develop potential diagnostic therapy and treatment on ESCC.

As a family of zinc-dependent proteolytic enzymes, the matrix metalloproteinases (MMPs) are able to degrade the extracellular matrix and basement membrane [5]. More and more studies have demonstrated the positive effects of MMPs on the tumor grow, neoangiogenesis, migration, and metastasis $[6,7]$. In the past two decades, several suppressors of MMPs have been developed in many types of tumors $[8,9]$. However, although in vitro and in vivo experiments are very beneficial, the clinical experiments failed due to the lack of susceptibility and serious adverse reactions. Many researchers have analyzed the possible reason, and several MMPs which exhibit tumor-suppressor functions may be the most important one $[10,11]$. With the developments of understating the potential function of MMPs in tumor progression, the sensitive narrow-spectrum MMPs inhibitors were currently being developed. In addition, some studies have reported the dysregulation of MMPs and their association with clinical outcome in several types of tumors [12-14]. 
In recent years, the effect of MMP12 has been verified in tumors. For example, MMP12 was highly expressed in adamantinomatous craniopharyngioma, while its knockdown inhibited the proliferation and attack of adamantinomatous craniopharyngioma cells [15]. Lin et al. reported that MMP12 was overexpressed in cervical cancer cells, and its silence clearly inhibited cell migration and invasion both in vitro and in vivo [16]. High expressions of MMP12 were related to the prognosis of several types of tumors, such as hepatocellular carcinoma and cutaneous melanoma $[17,18]$. However, the expression and function of MMP12 in ESCC were rarely reported.

This study is to sort out the clinical significance of MMP12 on ESCC and its contribution to cancer immunity.

\section{Methods and Materials}

2.1. Data Collection from the TCGA Database. The data of RNA transcriptome and the corresponding clinicopathological and survival for patients were obtained from the Cancer Genome Atlas (TCGA, https://cancergenome.nih. gov/). All assays were carried out based on the publication guidelines of TCGA. 160 ESCC samples and 11 nontumor samples were enrolled in this study.

2.2. Cell Culture and Transfection. Het-1A and ESCC cell lines (KYSE30, EC-1, Eca109 and EC9706) were bought from the Shanghai Institute of Biochemistry and Cell Biology (Shanghai, China). The cells were cultured in RPMI1640 medium supplemented with $10 \%$ fetal bovine serum (FBS) (Gibco BRL, USA) and maintained in a humidified incubator at $37^{\circ} \mathrm{C}$ with $5 \% \mathrm{CO}_{2}$.

MMP12 small interfering RNA (si-MMP12) and the corresponding control RNA (si-NC) were purchased from Jinlai Biology (Beijing, China). Lipofectamine 3000 was applied for cellular transfection.

2.3. Identification of Differently Expressed MMPs. "Limma" package of $\mathrm{R}$ was applied to sort out the differently expressed MMPs between ESCC specimens and nontumor specimens. The keys with $|\operatorname{logFC}| \geq 1$ and $p$ value $<0.05$ were defined as significant cutoff points. In addition, gene annotation and its data files of the differently expressed MMPs were collected through $\mathrm{R}$ software.

2.4. Prognosis-Related MMPs Screening. We constructed the Kaplan-Meier plots of MMPs in the TCGA dataset to comprehend the overall survival (OS) and verified it by logrank tests.

2.5. Screening of Dysregulated Genes and GO and KEGG Pathway Assays. We carried out GO and KEGG pathway assays on the dysregulated genes between high MMP12 expression group and low MMP12 expression group: GO assays included molecular function (MF), cell component (CC), and biological process (BP). KEGG (http://www. genome.jp/) was a novel method for exploring the related regulatory pathways involved in gene functions. ClusterProfiler package was applied for GO and KEGG pathway assays, while GOplot package was applied for cluster assays [19]. Besides, it was thought to grind a significant difference when both the $p$ value and $q$ value were less than 0.05 only.

2.6. Assessment of Immune Infiltration. As a deconvolution algorithm, CIBERSORT applied the expressions of 547 tag genes to define the structure of immune cells in specimens. Hence, the associated proportion of 22 infiltrating immune cells was examined by the use of CIBERSORT in all samples from TCGA datasets. $P<0.05$ was deem as statistically valuable.

2.7. Quantitative Real-Time PCR. RNA was isolated using TRIzol (Invitrogen, Pudong, Shanghai, China) following the manufacture's protocols. A Transcript RT kit (Vazyme, Nanjing, Jiangsu, China) was applied to compound the first strand cDNA. Real-time RT-PCR was performed to detect the expression of CRNDE using the One-Step SYBR PrimeScript RT-PCR Kit (Takara). GADPH was used as endogenous controls. The relative expressions were calculated using the $2^{-\Delta \Delta \mathrm{Ct}}$ method.

2.8. CCK-8 Assays. Cellular proliferation was examined applying the Cell Counting Kit-8 (Beyotime, Haidian, China). Cells were seeded into 48 -well plates at $3 \times 10^{3}$ cells/ well cell concentration. Then, $15 \mu \mathrm{L}$ CCK-8 solution was added to each well. At a wavelength of $450 \mathrm{~nm}$ for each well, the absorbance was examined.

2.9. Statistical Analysis. We adopted R (version 3.6.0) to conduct statistical analyses. The Wilcox test was applied to determine the dysregulated genes and infiltrative immune cells. We obtain the survival curves by the Kaplan-Meier method and compared by the log-rank test. $P$ value $<0.05$ was considered statistically significant.

\section{Results}

3.1. Identification of the Dysregulated MMPs in ESCC. To screen the dysregulated MMPs in ESCC, we analyzed TCGA datasets using Limma and edgeR packages. The dysregulated MMPs were shown in heat map (Figure 1(a)). We noticed that the behaviors of MMP12 were distinctly enhanced on ESCC specimens comparing to nontumor specimens, including MMP1 (Figure 1(b)), MMP3 (Figure 1(c)), MMP7 (Figure 1(d)), MMP8 (Figure 1(e)), MMP9 (Figure 1(f)), MMP13 (Figure 1(g)), MMP10 (Figure 1(h)), MMP11 (Figure 1(i)), MMP12 (Figure 1(j)), MMP14 (Figure 1(k)), MMP17 (Figure 1(l)), and MMP20 (Figure 1(m)).

3.2. The Survival-Related MMPs in ESCC. Then, we performed Kaplan-Meier methods to screen survival-related MMPs in ESCC. Only high MMP12 expression was associated with a short overall survival of ESCC patients (Figure 2(a)). For other MMPs, the results indicated no 


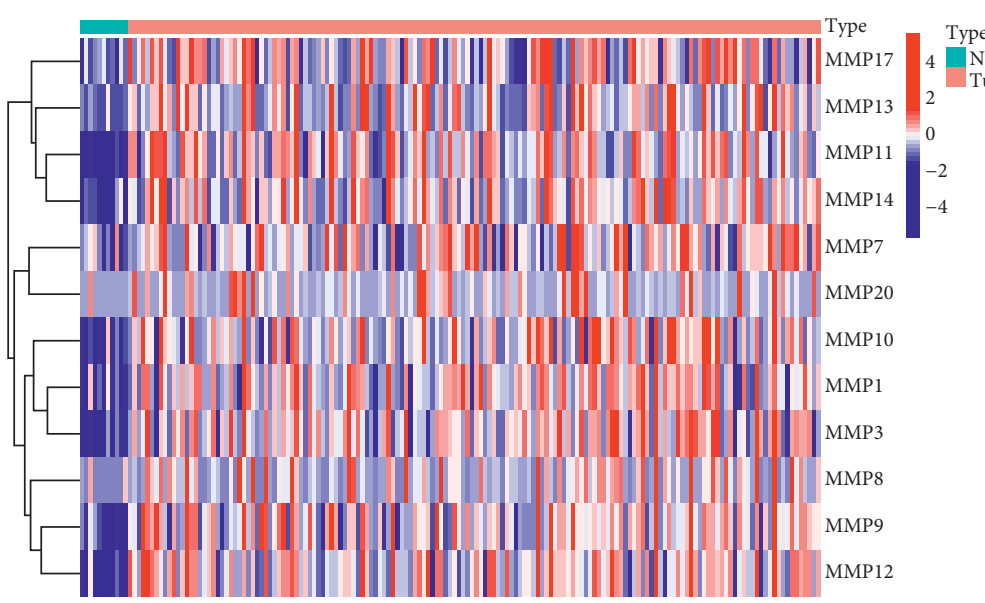

(a)

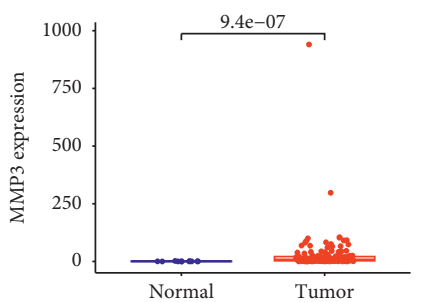

Type

Eormal

Tumor

(c)

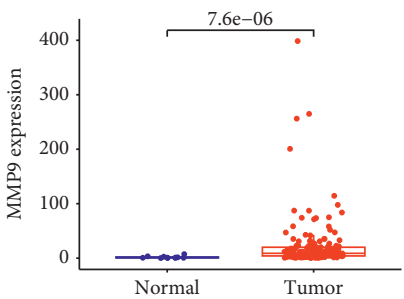

Type

由rmal

$\bullet$ Tumor

(f)

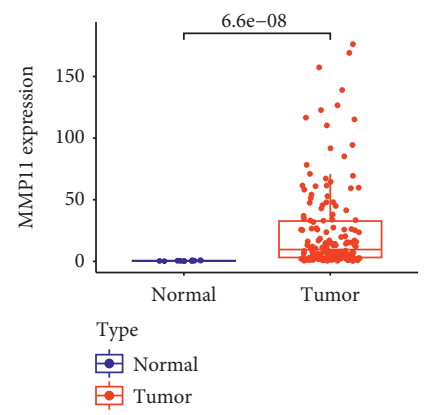

(i)

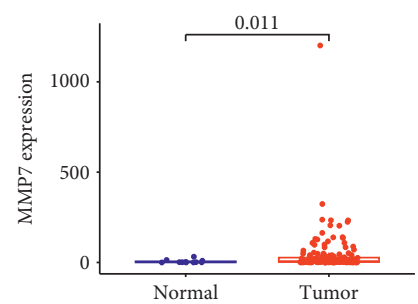

Type

- Normal

Tumor

(d)

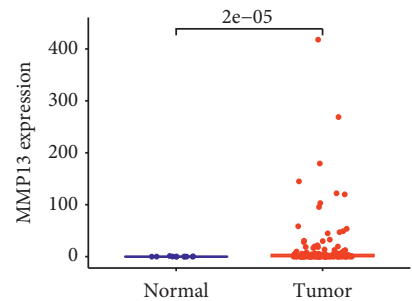

Type

Normal

Tumor

(g)

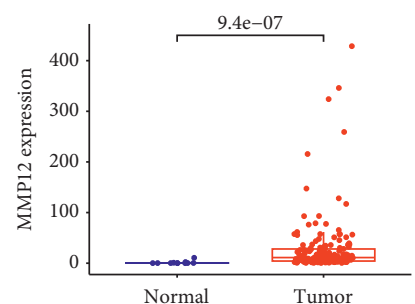

Type

-

Tumor

(j)

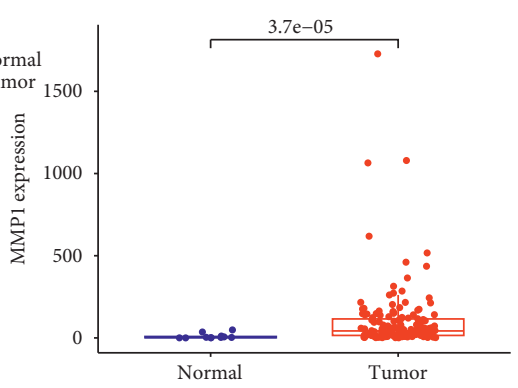

Type

宜 Normal

审 Tumor

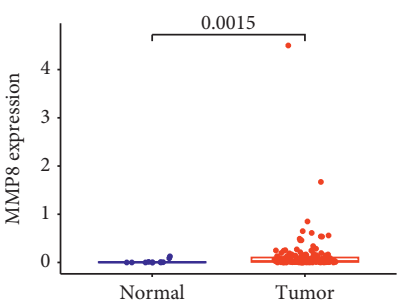

Type

Normal Tumor

(e)

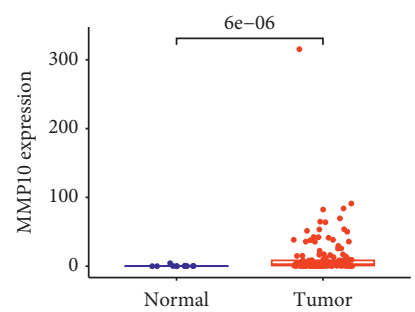

Type

Normal

Tumor

(h)

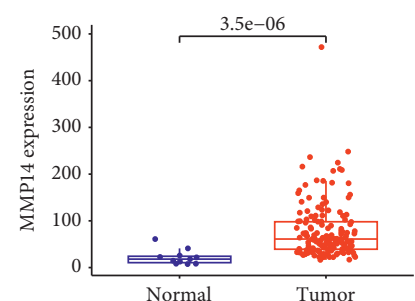

Type

E Normal

Tumor

(k)

Figure 1: Continued. 


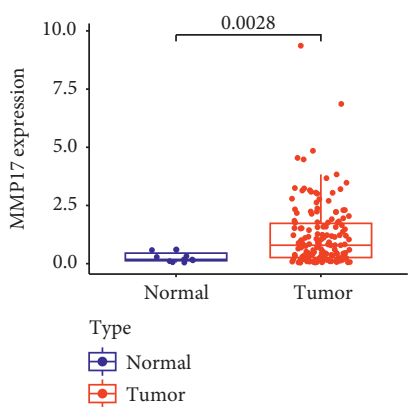

(1)

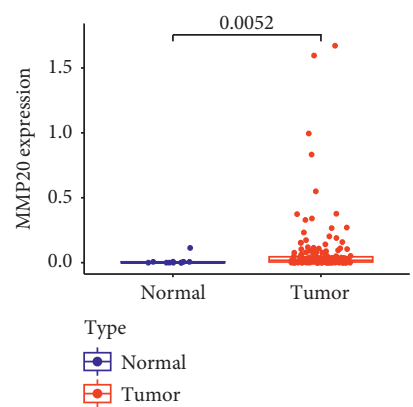

(m)

FIGURE 1: Identification of the dysregulated MMPs in ESCC. (a) Hierarchical clustering analysis of differently expressed MMPs in ESCC and normal tissues. (b)-(m) An increased expression of (b) MMP1, (c) MMP3, (d) MMP7, (e) MMP8, (f) MMP9, (g) MMP13, (h) MMP10, (i) MMP11, (j) MMP12, (k) MMP14, (l) MMP17, and (m) MMP20 observed in ESCC specimens compared with nontumor specimens.

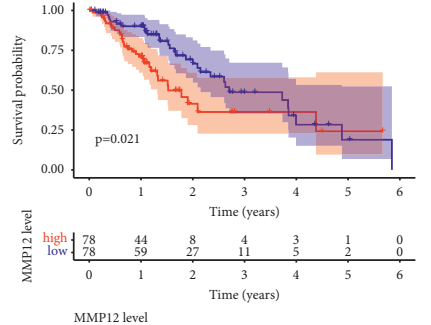

high
L low

(a)

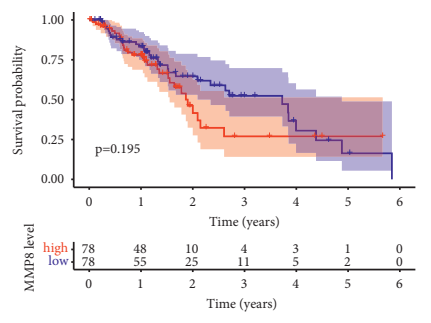
MMPs leve
N high
= low

(e)

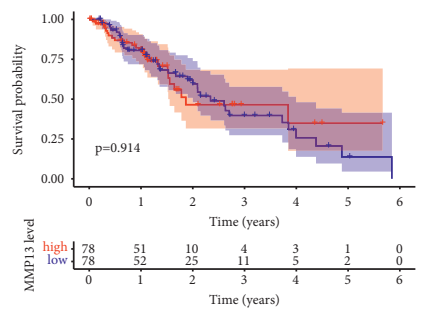

MMP1 3 level Ehigh
hew
low

(i)
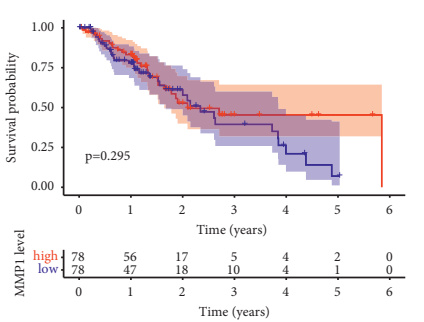

$\underset{\text { MMP level }}{\text { high }}$

high

(b)

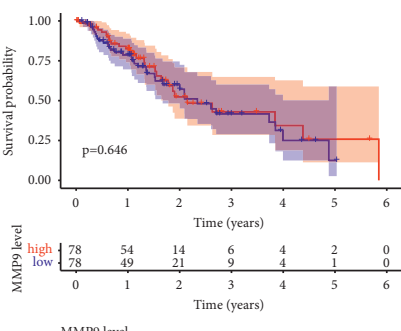

MMP9 leve
E high
a low

(f)

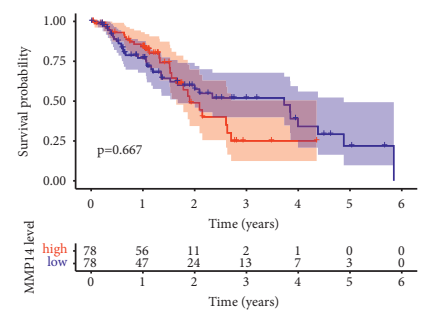

MMP1 4 leve
Ehigh
Elow
$=$

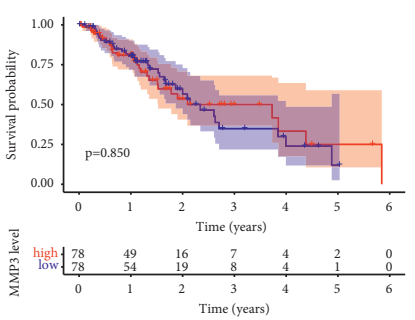

MMP3 level
thigh

high
Ilow
low

(c)

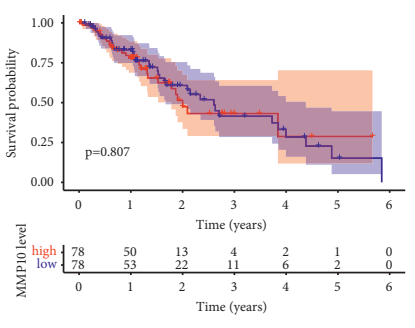

MMP10 level
E high

E high

(g)

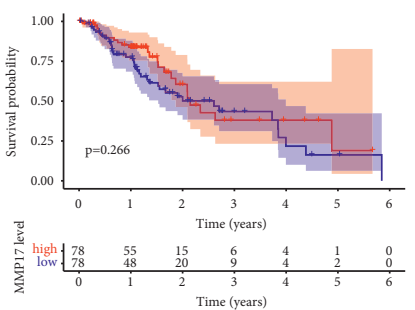

MMP17 leve
E high
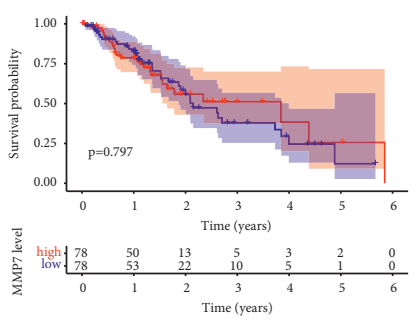

MMP7 level high
\pm low

(d)
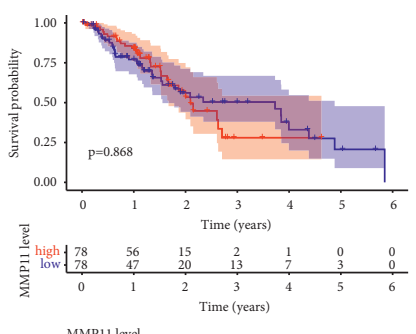

MMP1 l leve
D high
Elow

(h)
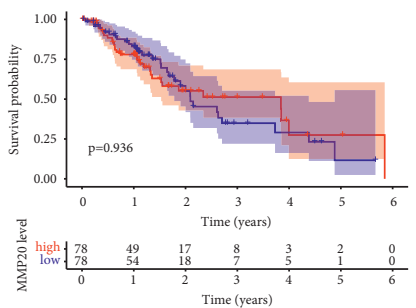

MMP20 level

Shigh
L low
low

(j)

(1)

Figure 2: Identification of survival-related MMPs. (a) Survival analysis for MMP12 in ESCC. Patient with high MMP12 expression showed a shorter overall survival of ESCC patients. (b)-(l) Survival analysis for (b) MMP1, (c) MMP3, (d) MMP7, (e) MMP8, (f) MMP9, (g) MMP10, (h) MMP11, (i) MMP13, (j) MMP14, (k) MMP17, and (l) MMP20 in ESCC. According to median expression of MMPs, the patients were classified into high-level and low-level groups.

obvious difference in the survival rate between patients with high MMPs and low ones (Figures 2(b)-2(l)). Thus, our attention focused on MMP12.
3.3. Correlation between MMP12 Behaviors and Clinical Trials in ESCC Patients. We further examined the associations between MMP12 patients' clinical features and the 


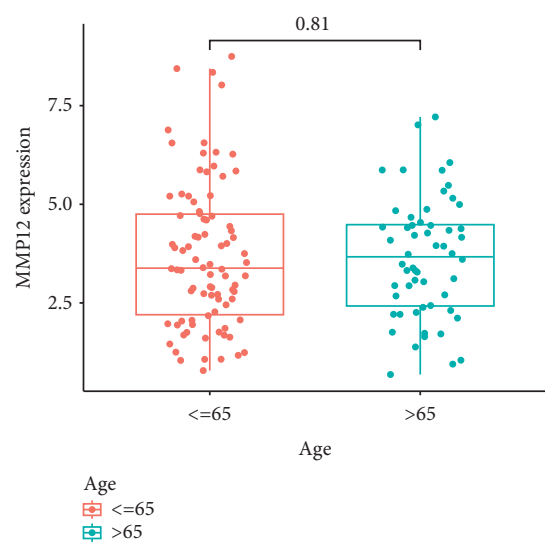

(a)

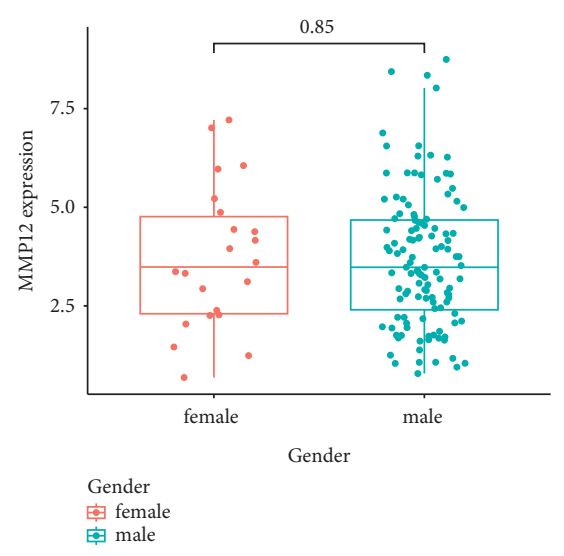

(b)

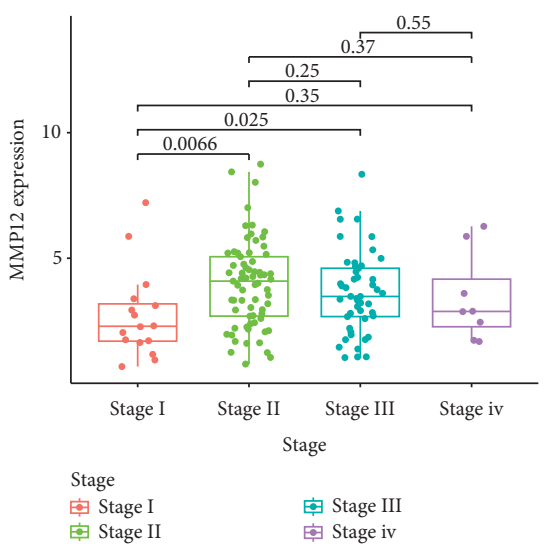

(c)

FIgURE 3: The relationship between MMP12 and clinical features. (a) Age $(p=0.81)$. (b) Gender $(p=0.85)$. (c) Clinical phase ( $p<0.05)$.

MMP12 expressions and concluded that MMP12 expressions were not associated with age $(p=0.81$, Figure $3(\mathrm{a}))$ and gender $(p=0.85$, Figure $3(\mathrm{~b}))$. However, we observed that the expressions of MMP12 in ESCC were distinctly linked to stage (Figure 3(c)).

3.4. Functional Enrichment Analysis of Genes That Were Coexpressed with MMP12. To explore the biological function of MMP12 in ESCC, we divided all ESCC specimens into two (high and low) based on the mean expression of MMP12 in all ESCC samples. A total of 15 differently expressed genes between low and high MMP12 expression groups were screened. Next, 15 genes were chosen to perform GO and KEGG analyses using the ClusterProfiler $\mathrm{R}$ package. The results showed that MMP12-associated dysregulated genes were mainly involved in processes like the collagen catabolic process, extracellular matrix disassembly, collagen-containing, blood microparticle, metalloendopeptidase activity, and chemokine activity (Figure 4(a)). Meanwhile, KEGG pathway analysis showed that pathways were significantly enriched (Figure 4(b)) including the relaxin signaling pathway, Toll-like receptor signaling pathway, TNF signaling pathway, IL-17 signaling pathway, and transcriptional misregulation in cancer [20-22].

3.5. Distribution of Tumor-Infiltrating Immune Cells. We explored the pattern of immune cells by the use of the CIBERSORT method. Its composition on ESCC samples and the associations among immune cells are shown in Figures 5(a) and 5(b), respectively. However, we found that there were no significant differences in the levels of tumorinfiltrating immune cells between tumor and nontumor specimens (Figures 6(a) and 6(b)). Several studies had proved that immune cells might serve as independent indicators of survivals and immunotherapy efficacies in ESCC $[23,24]$. Then, we needed to finalize whether MMP12 behaviors were associated with immune cells. Importantly, we observed that the levels of MMP12 were in positive association with the infiltrated levels of mast cells activated
(Figure 7(a)) and macrophages M0 (Figure 7(b)). However, eosinophils (Figure $7(\mathrm{c})$ ), B cells naïve (Figure $7(\mathrm{~d})$ ), and mast cells resting (Figure 7(e)) exhibited an opposite result.

3.6. The Oncogenic Roles of MMP12 in ESCC Progression. To demonstrate the expression of MMP12 in ESCC, we performed RT-PCR using four ESCC cell lines and observed that MMP12 expression was distinctly increased in four ESCC cell lines compared with Het-1A cells (Figure 8(a)). Given that Eca109 and EC-1 exhibited a relatively higher level among four ESCC cells, we chose them for further study. We used loss-of-function experiments to explore the possible effects of MMP12 in ESCC. The interference efficiencies of siRNA are shown in Figure 8(b), suggesting that siRNA efficiently decreased MMP12 expressions. CCK-8 assays revealed that Eca109 and EC-1 proliferation was distinctly suppressed when silencing MMP12 (Figures 8(c) and $8(\mathrm{~d})$ ).

\section{Discussion}

The research for effective molecular markers for diagnosis and prognosis of ESCC is very important for prognosis of patients [25]. In the last decade, more and more tumorrelated genes have been well studied. For instance, as a main RNA N6-adenosine methyltransferase, METTL3 was highly expressed in gastric cancer. Clinical assays disclosed that overexpression of METTL3 predicted a poor outcome of gastric cancer patients [26]. Hu et al. outlined that HIF- $1 \alpha$ was distinctly enhanced on ESCC and was in line with metastasis, recurrence, and poor prognosis. Functionally, knockdown of HIF- $1 \alpha$ suppressed the metastasis of ESCC cells via targeting SP1 [27]. These findings encouraged us to further identify functional genes involved in ESCC progression.

MMPs are commonly expressed in normal specimens [5]. It has been demonstrated that the expressions and activities exhibited an increased trend during inflammation and tumor progression $[28,29]$. In this study, we analyzed the expressing pattern of MMPs in ESCC specimens based 


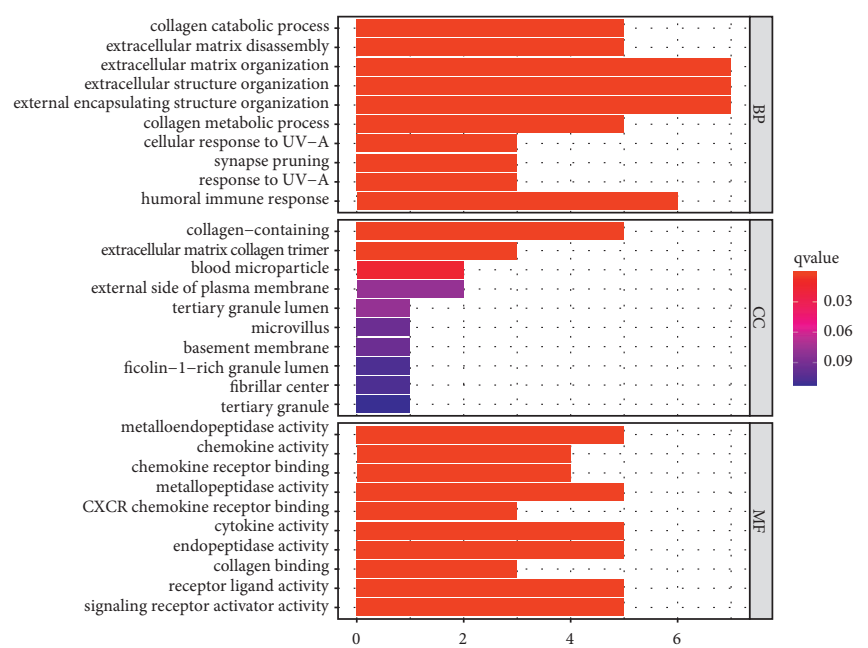

(a)

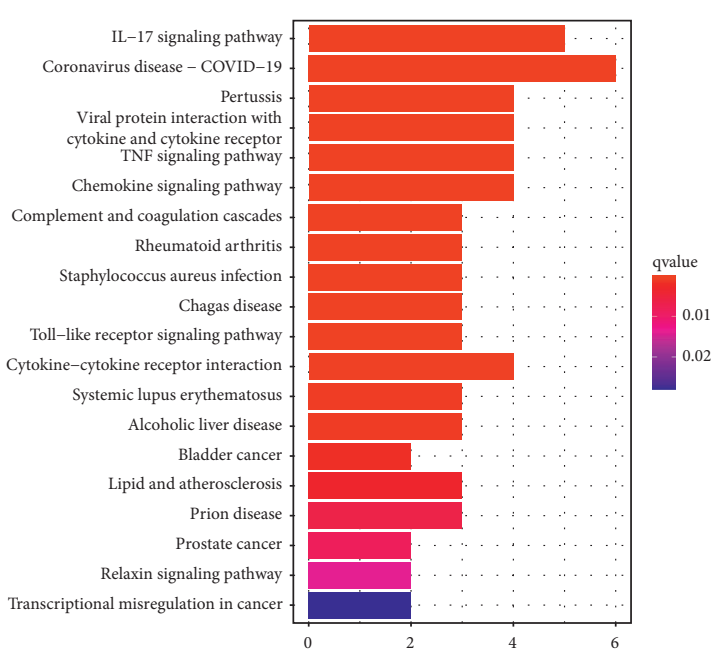

(b)

FIGURE 4: Functional enrichment analysis of the dysregulated genes in low and high MMP12 expression groups. (a) The enriched GO annotation included the biological process, cellular component, and molecular function. (b) Dotplot of the KEGG signal pathway.

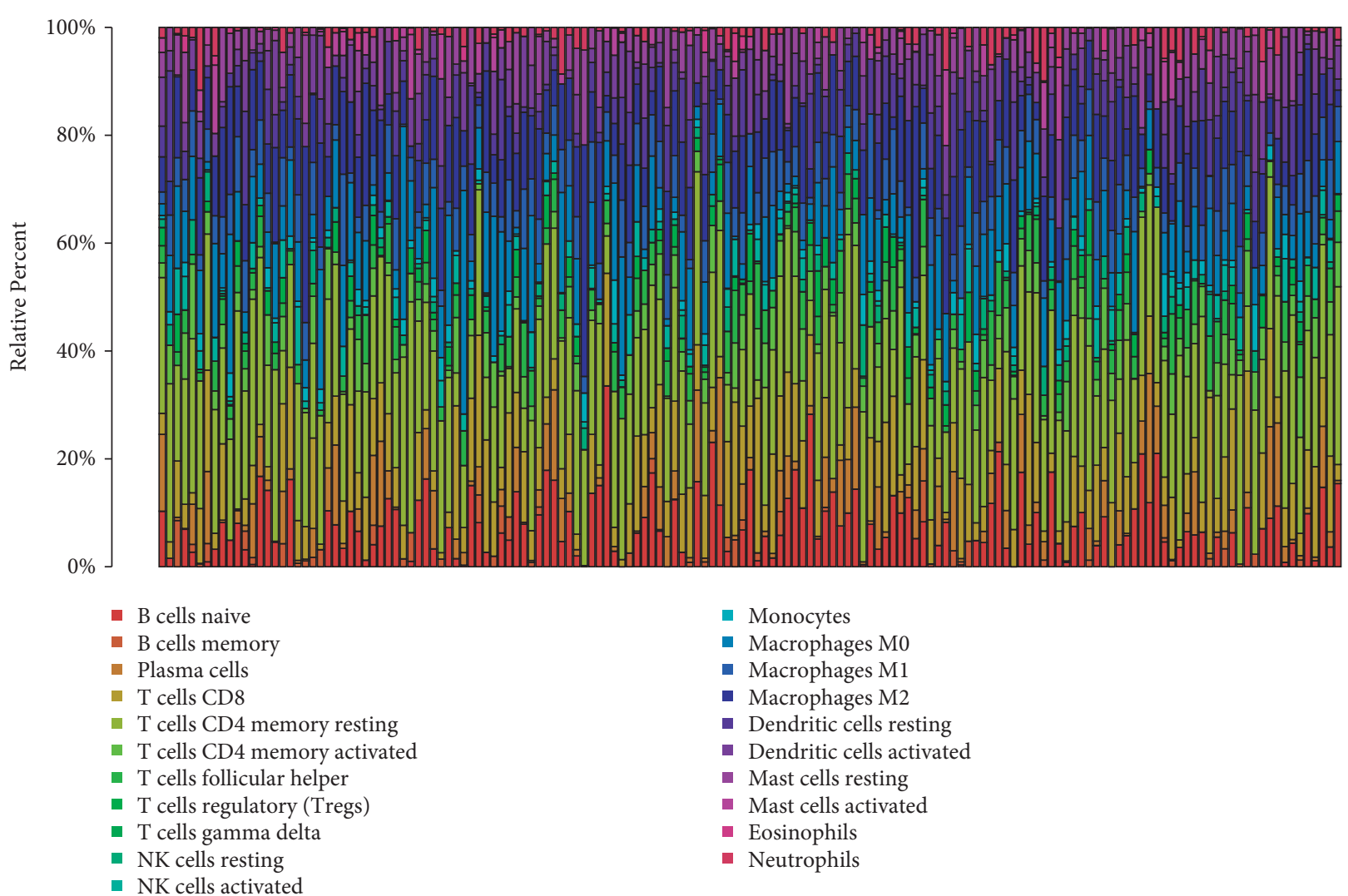

(a)

Figure 5: Continued. 


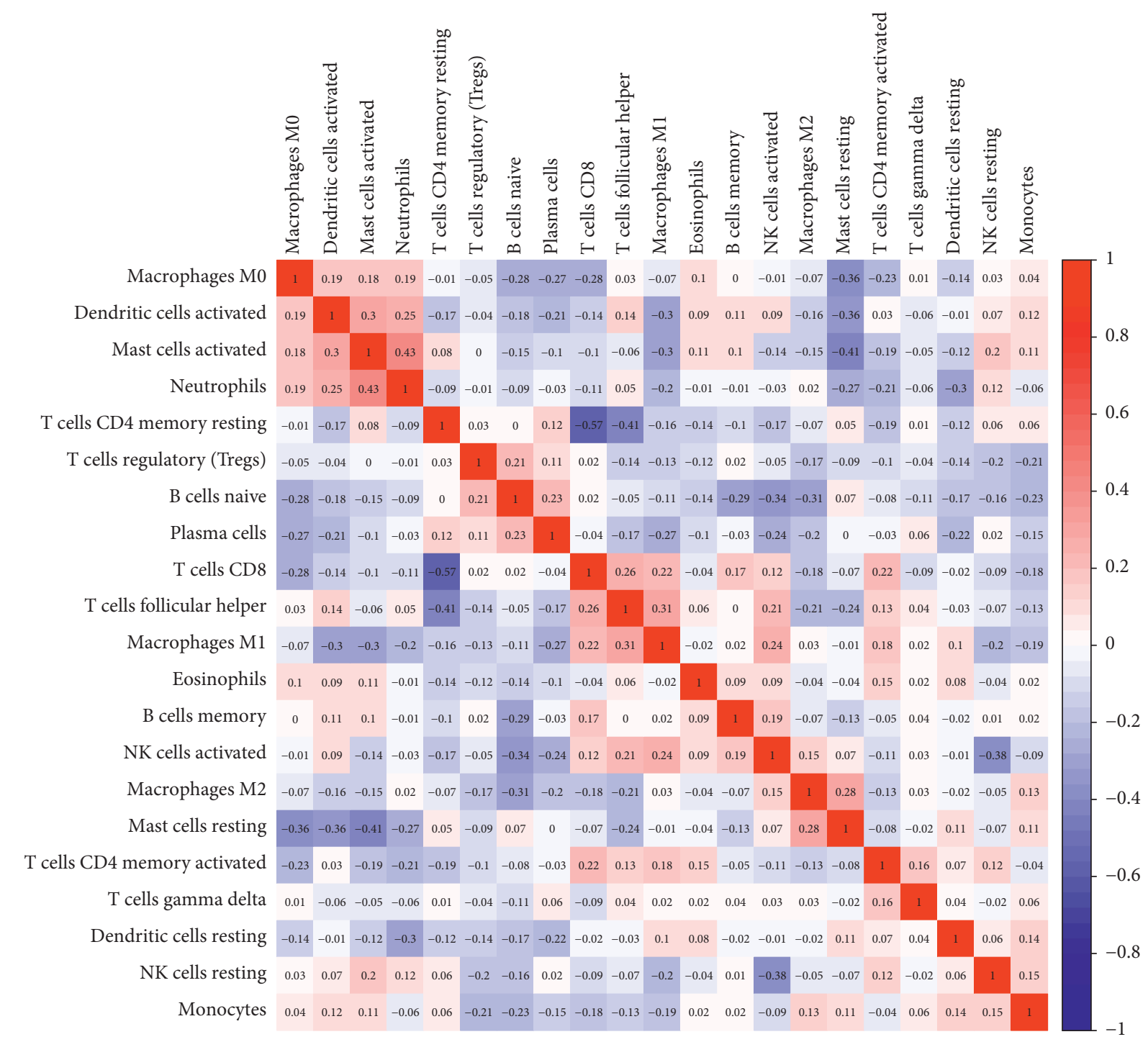

(b)

Figure 5: (a), (b) The proportion of the 22 immune cells detected by the CIBERSORT algorithm.

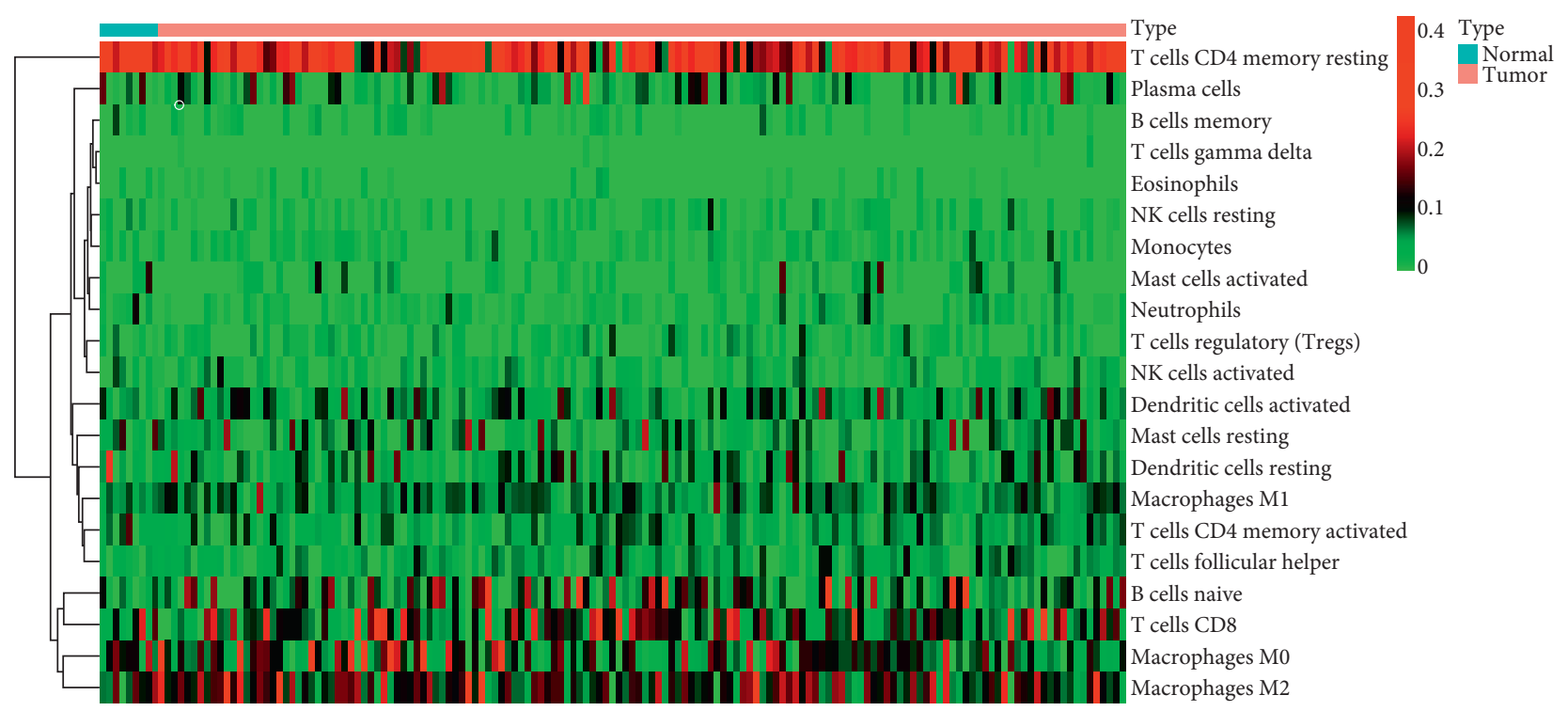

(a)

Figure 6: Continued. 


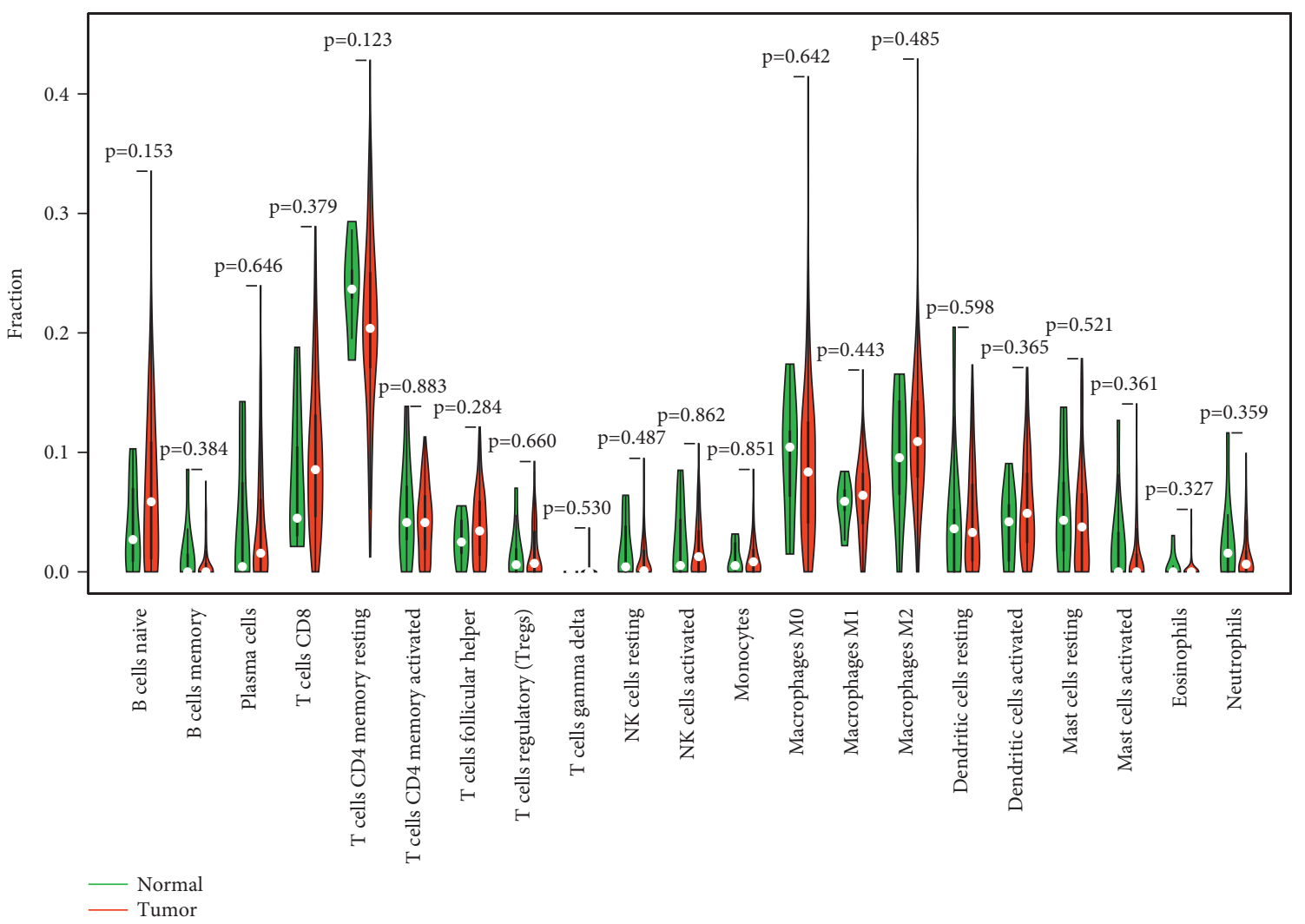

(b)

FIgURE 6: Analysis of the levels of 22 TIICs and its correlations in ESCC specimens and 11 normal cases. (a) Heatmaps indicated the expressing pattern of the immune cell between ESCC specimens and nontumor specimens. (b) The differences in the structure of TIICs between normal tissue and ESCC tissues.

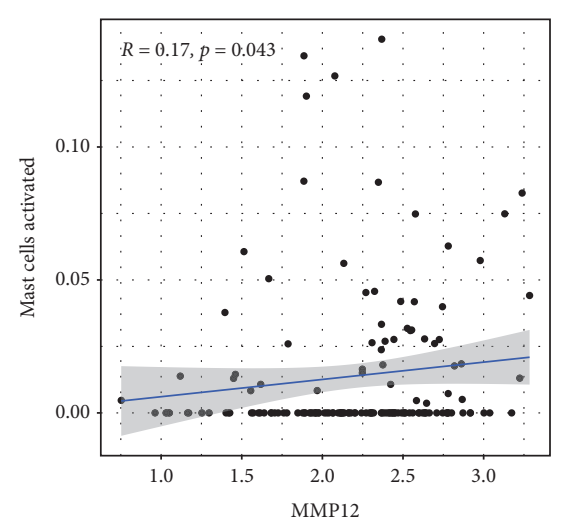

(a)

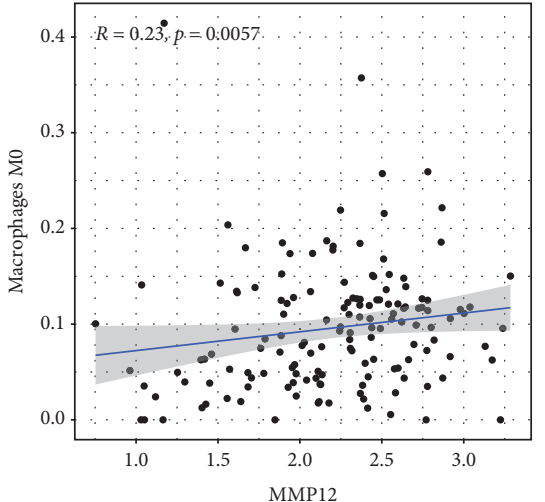

(b)

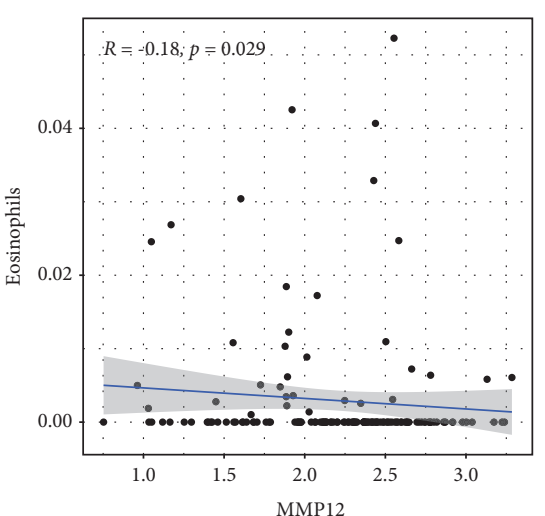

(c)

Figure 7: Continued. 


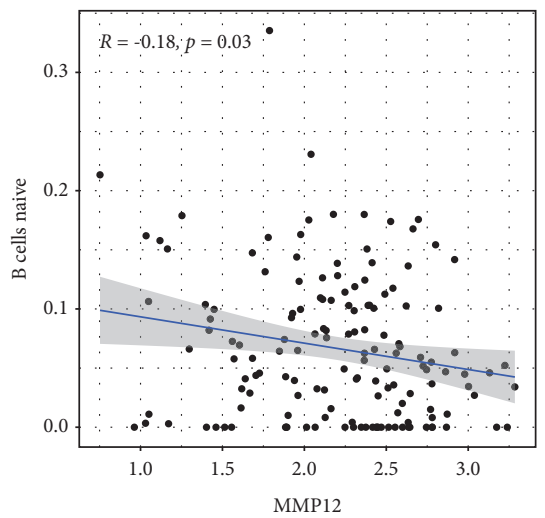

(d)

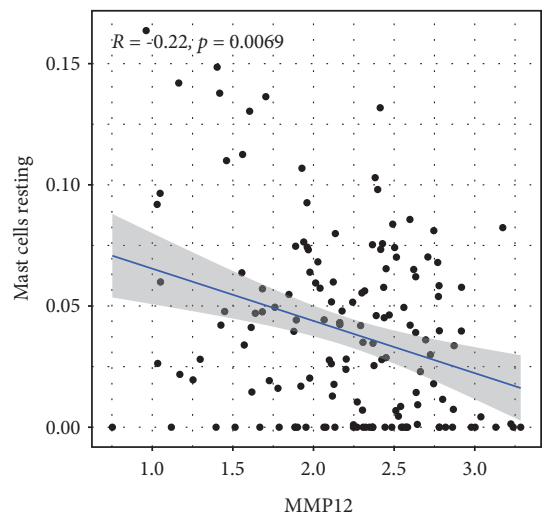

(e)

Figure 7: The correlation between MMP12 and immune infiltration level in ESCC. (a), (b) A positive association between MMP12 behaviors and the levels of macrophages M0 and mast cells activated was observed. (c)-(e) A negative association between MMP12 expression and the levels of B cells naïve, eosinophils, and mast cells resting was observed.

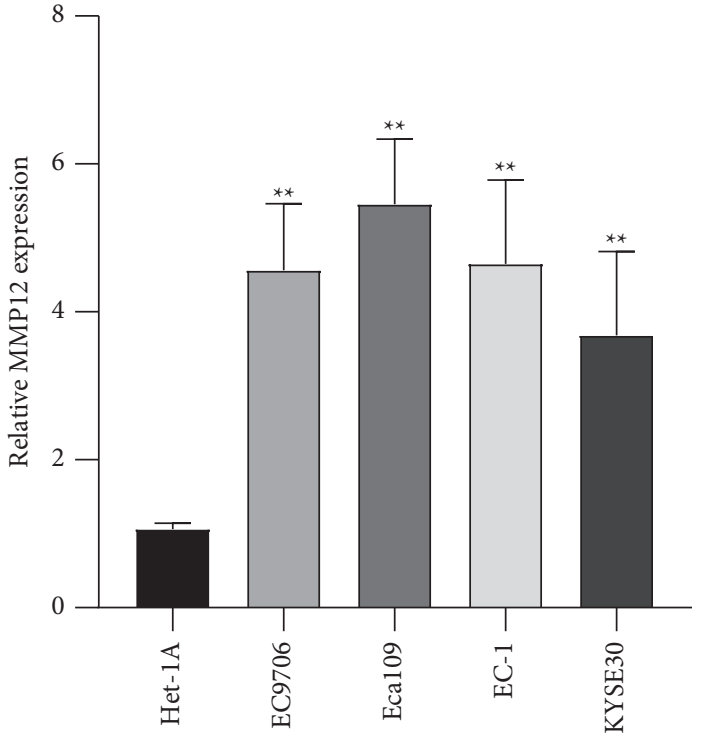

(a)

Eca109

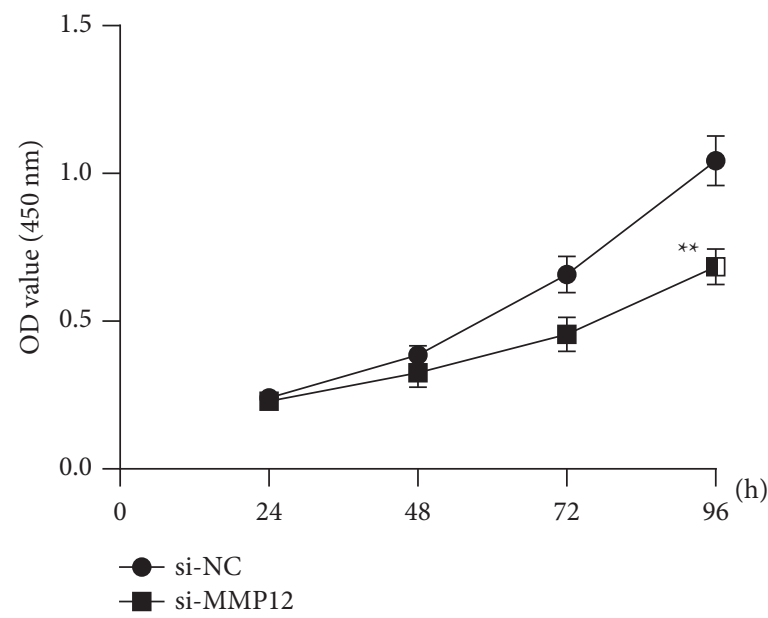

(c)

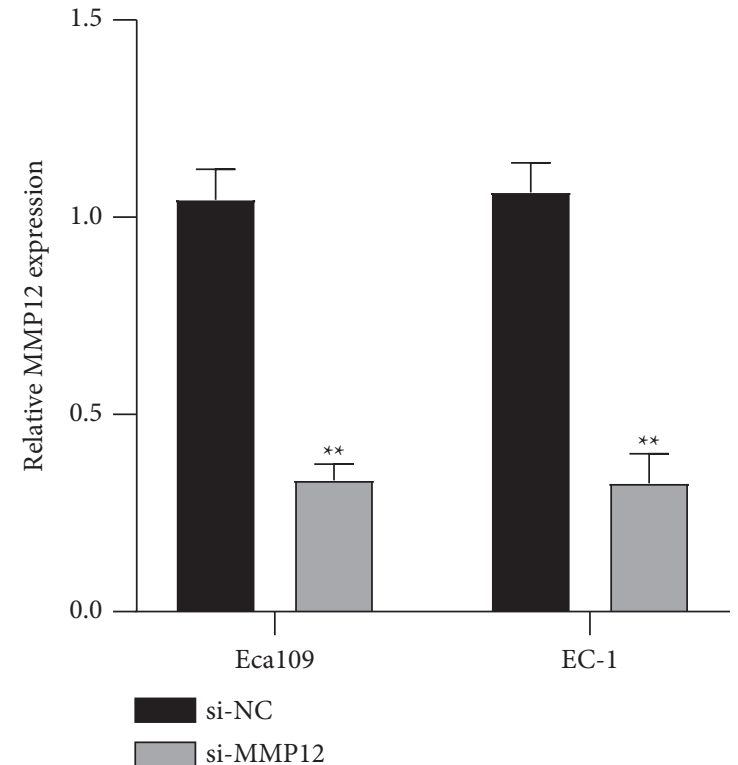

(b)

EC-1

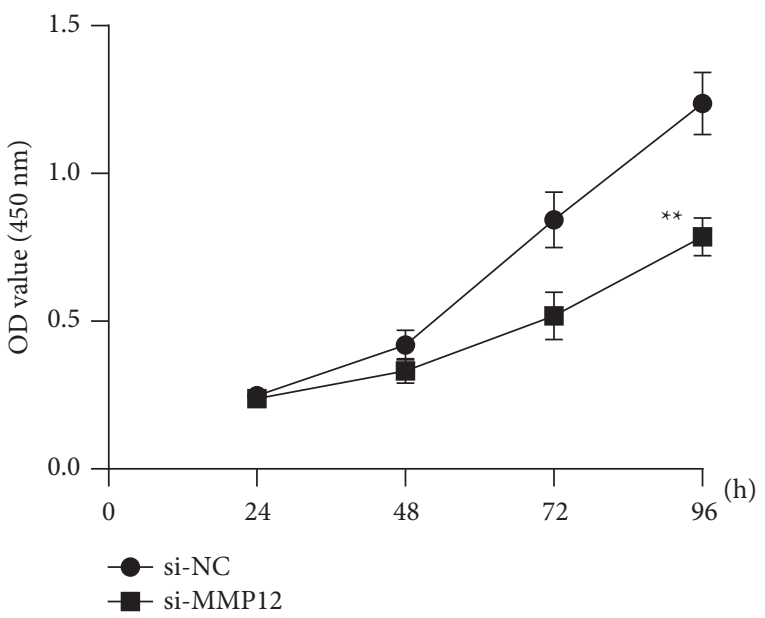

(d)

FIGURE 8: Knockdown of MMP12 suppressed the proliferation of ESCC cells. (a) RT-PCR for the expression of MMP12 in ESCC cell lines. (b) The expression of MMP12 decreased in Eca109 and EC-1 cells transfected with si-MMP12. (c), (d) CCK-8 assay indicated that OD values of Eca109 and EC-1 cells significantly decreased when transfected with si-MMP12. ${ }^{* *} P<0.01$. 
on TCGA datasets and identified 12 dysregulated MMPs in ESCC, including MMP1, MMP12, MMP20, MMP17, MMP14, MMP11, MMP10, MMP13, MMP9, MMP8, MMP7, and MMP3. Among the above genes, only MMP12 was associated with five-year survival of ESCC patients, and its high expression was also associated with advanced clinical stages in ESCC specimens. To explore the possible function of MMP12 in ESCC progression, we performed KEGG assays, which revealed that the genes associated with MMP12 were mainly enriched in several tumor-related pathways including PI3K-Akt signaling, estrogen signaling, and relaxin signaling [30-32]. Moreover, we also proved that knockdown of MMP12 distinctly suppressed the proliferation of ESCC cells. Besides, the effect of MMP12 has been reported in several tumors. For instance, MMP12 was highly expressed in lung adenocarcinoma, and its knockdown distinctly inhibited the growth and invasion of lung adenocarcinoma cells [33]. In hepatocellular carcinoma, high MMP12 expression predicted a poor prognosis. Importantly, the prognostic value of MMP12 was also demonstrated in 93 patients, which was consistent with our findings [34]. Thus, together with previous findings, these results indicated MMP12 as a novel prognostic biomarker for ESCC patients.

Several antibodies targeting immune checkpoints for the treatments of ESCC were approved by the FDA for the sake of its efficacy $[35,36]$. Biomarkers would be tremendously valuable in improving therapeutic decision making in ESCC [37]. In this study, we elaborated on the impact of MMP12 on the immune systems. However, we did not observe a distinct different of the level of immune cells between ESCC specimens and nontumor specimens. Then, we needed to figure out whether MMP12 expression was related to immune cell on ESCC and observed that upregulated MMP12 was positively in line with the infiltration levels of macrophages M0 and mast cells activated. In addition, downregulated MMP12 was negatively correlated with the infiltration of B cells naïve, eosinophils, and mast cells resting. Our findings provided evidence that MMP12 may be a potential immunotherapeutic object for ESCC.

There are some limitations in our study. The first one is the limited sample size that needs to be improved. The second one is the lack of the exploration of mechanisms underpinning MMP12-medicated tumor immunity and the prognostic values of immune signatures. The third one is that examining the biomarkers in the serum/plasma samples might help monitor the therapy response in realtime.

\section{Conclusion}

We identified several dysregulated MMPs in ESCC, and their function needed to be further studied. We provided clinical evidence that MMP12 was highly expressed in ESCC and can serve as an independent prognostic marker for survival in ESCC. Our conclusion is that MMP12 might play a role in controlling the tumor immune microenvironments. Additional investigation is required to confirm the findings before the clinical application of MMP12.

\section{Data Availability}

The analyzed datasets generated during the study are available from the corresponding author upon request.

\section{Disclosure}

Jing-tao Mao, Qiang Lu, and Peng-yu Jing are the co-first authors.

\section{Conflicts of Interest}

The authors declare that they have no conflicts of interest.

\section{Authors' Contributions}

Jing-tao Mao, Qiang Lu, and Peng-yu Jing contributed equally to this work.

\section{Acknowledgments}

This work was supported by the Youth Program of the National Natural Science Foundation of China (82002422).

\section{References}

[1] A. Pennathur, M. K. Gibson, B. A. Jobe, and J. D. Luketich, "Oesophageal carcinoma," Lancet, vol. 381, no. 9864, pp. 400-412, 2013.

[2] C. C. Abnet, M. Arnold, and W.-Q. Wei, "Epidemiology of esophageal squamous cell carcinoma," Gastroenterology, vol. 154 , no. 2, pp. 360-373, 2018.

[3] Z. W. Reichenbach, M. G. Murray, R. Saxena et al., "Clinical and translational advances in esophageal squamous cell carcinoma," Advances in Cancer Research, vol. 144, pp. 95135, 2019.

[4] M. di Pietro, M. I. Canto, and R. C. Fitzgerald, "Endoscopic management of early adenocarcinoma and squamous cell carcinoma of the esophagus: screening, diagnosis, and therapy," Gastroenterology, vol. 154, no. 2, pp. 421-436, 2018.

[5] C. Kapoor, S. Vaidya, V. Wadhwan, G. Kaur, Hitesh, and A. Pathak, "Seesaw of matrix metalloproteinases (MMPs)," Journal of Cancer Research and Therapeutics, vol. 12, no. 1, pp. 28-35, 2016.

[6] E.-S. E. Mehana, A. F. Khafaga, and S. S. El-Blehi, “The role of matrix metalloproteinases in osteoarthritis pathogenesis: an updated review," Life Sciences, vol. 234, Article ID 116786, 2019.

[7] K. Kessenbrock, V. Plaks, and Z. Werb, "Matrix metalloproteinases: regulators of the tumor microenvironment," Cell, vol. 141, no. 1, pp. 52-67, 2010.

[8] J. Gonzalez-Molina, S. Gramolelli, Z. Liao, J. W. Carlson, P. M. Ojala, and K. Lehti, "MMP14 in sarcoma: a regulator of tumor microenvironment communication in connective tissues," Cells, vol. 8, no. 9, 2019.

[9] C. Joseph, M. Alsaleem, N. Orah et al., "Elevated MMP9 expression in breast cancer is a predictor of shorter patient survival," Breast Cancer Research and Treatment, vol. 182, no. 2, pp. 267-282, 2020.

[10] C. Lombard, J. Saulnier, and J. Wallach, "Assays of matrix metalloproteinases (MMPs) activities: a review," Biochimie, vol. 87, no. 3-4, pp. 265-272, 2005. 
[11] M. Egeblad and Z. Werb, "New functions for the matrix metalloproteinases in cancer progression," Nature Reviews Cancer, vol. 2, no. 3, pp. 161-174, 2002.

[12] C. Böckelman, I. Beilmann-Lehtonen, T. Kaprio et al., "Serum MMP-8 and TIMP-1 predict prognosis in colorectal cancer," $B M C$ Cancer, vol. 18, no. 1, p. 679, 2018.

[13] J. Ren, J. Liu, and X. Sui, "Correlation of COX-2 and MMP-13 expressions with gastric cancer and their effects on prognosis," Journal of B.U.ON.: Official Journal of the Balkan Union of Oncology, vol. 23, pp. 665-671, 2018.

[14] X. Gou, H. Chen, F. Jin et al., "Expressions of CD147, MMP-2 and MMP-9 in laryngeal carcinoma and its correlation with poor prognosis," Pathology and Oncology Research, vol. 20, no. 2, pp. 475-481, 2014.

[15] M. Li, L. Zhou, S. Li et al., "MMP12 is a potential therapeutic target for Adamantinomatous craniopharyngioma: conclusions from bioinformatics analysis and in vitro experiments," Oncology Letters, vol. 22, no. 1, p. 536, 2021.

[16] C.-L. Lin, T.-H. Ying, S.-F. Yang et al., "MTA2 silencing attenuates the metastatic potential of cervical cancer cells by inhibiting AP1-mediated MMP12 expression via the ASK1/ MEK3/p38/YB1 axis," Cell Death \& Disease, vol. 12, no. 5, p. $451,2021$.

[17] H. Gao, X. Zhou, H. Li et al., "Role of matrix metallopeptidase 12 in the development of hepatocellular carcinoma," Journal of Investigative Surgery, vol. 34, no. 4, pp. 366-372, 2021.

[18] Z. Zhang, S. Zhu, Y. Yang, X. Ma, and S. Guo, "Matrix metalloproteinase-12 expression is increased in cutaneous melanoma and associated with tumor aggressiveness," Tumor Biology, vol. 36, no. 11, pp. 8593-8600, 2015.

[19] G. Yu, L.-G. Wang, Y. Han, and Q.-Y. He, "clusterProfiler: an $\mathrm{R}$ package for comparing biological themes among gene clusters," OMICS: A Journal of Integrative Biology, vol. 16, no. 5, pp. 284-287, 2012.

[20] G. Chen and D. V. Goeddel, "TNF-R1 signaling: a beautiful pathway," Science, vol. 296, no. 5573, pp. 1634-1635, 2002.

[21] M. J. McGeachy, D. J. Cua, and S. L. Gaffen, "The IL-17 family of cytokines in health and disease," Immunity, vol. 50, no. 4, pp. 892-906, 2019.

[22] K.-H. Lim and L. M. Staudt, "Toll-like receptor signaling," Cold Spring Harbor Perspectives in Biology, vol. 5, no. 1, Article ID a011247, 2013.

[23] R. L. Sabado, S. Balan, and N. Bhardwaj, "Dendritic cell-based immunotherapy," Cell Research, vol. 27, no. 1, pp. 74-95, 2017.

[24] B. J. Laidlaw, J. E. Craft, and S. M. Kaech, "The multifaceted role of CD4+ T cells in CD8+ T cell memory," Nature Reviews Immunology, vol. 16, no. 2, pp. 102-111, 2016.

[25] Y.-M. Yang, P. Hong, W. W. Xu, Q.-Y. He, and B. Li, "Advances in targeted therapy for esophageal cancer," Signal Transduction and Targeted Therapy, vol. 5, no. 1, p. 229, 2020.

[26] B. Yue, C. Song, L. Yang et al., "METTL3-mediated N6methyladenosine modification is critical for epithelial-mesenchymal transition and metastasis of gastric cancer," Molecular Cancer, vol. 18, no. 1, p. 142, 2019.

[27] X. Zhao, Y. P. Tang, C. Y. Wang, J. X. Wu, and F. Ye, "Prognostic values of STAT3 and HIF- $1 \alpha$ in esophageal squamous cell carcinoma," European Review for Medical and Pharmacological Sciences, vol. 23, no. 8, pp. 3351-3357, 2019.

[28] G. Gonzalez-Avila, B. Sommer, A. A. García-Hernández, and C. Ramos, "Matrix metalloproteinases' role in tumor microenvironment," Advances in Experimental Medicine \& Biology, vol. 1245, pp. 97-131, 2020.
[29] J. Xiong and H. Gao, "Matrix metalloproteases-responsive nanomaterials for tumor targeting diagnosis and treatment," Journal of Microencapsulation, vol. 34, no. 5, pp. 440-453, 2017.

[30] M. Martini, M. C. De Santis, L. Braccini, F. Gulluni, and E. Hirsch, "PI3K/AKT signaling pathway and cancer: an updated review," Annals of Medicine, vol. 46, no. 6, pp. 372-383, 2014.

[31] E. Kulkoyluoglu-Cotul, A. Arca, and Z. Madak-Erdogan, "Crosstalk between estrogen signaling and breast cancer metabolism," Trends in Endocrinology and Metabolism, vol. 30, no. 1, pp. 25-38, 2019.

[32] T. Thanasupawat, A. Glogowska, S. Nivedita-Krishnan, B. Wilson, T. Klonisch, and S. Hombach-Klonisch, "Emerging roles for the relaxin/RXFP1 system in cancer therapy," Molecular and Cellular Endocrinology, vol. 487, pp. 85-93, 2019.

[33] F.-Z. Lv, J.-L. Wang, Y. Wu, H.-F. Chen, and X.-Y. Shen, "Knockdown of MMP12 inhibits the growth and invasion of lung adenocarcinoma cells," International Journal of Immunopathology \& Pharmacology, vol. 28, no. 1, pp. 77-84, 2015.

[34] M. K. He, Y. Le, Y. F. Zhang et al., "Matrix metalloproteinase 12 expression is associated with tumor FOXP3(+) regulatory $\mathrm{T}$ cell infiltration and poor prognosis in hepatocellular carcinoma," Oncology Letters, vol. 16, pp. 475-482, 2018.

[35] Y. Zheng, Z. Chen, Y. Han et al., "Immune suppressive landscape in the human esophageal squamous cell carcinoma microenvironment," Nature Communications, vol. 11, no. 1, p. $6268,2020$.

[36] J. Yang, X. Liu, S. Cao, X. Dong, S. Rao, and K. Cai, "Understanding esophageal cancer: the challenges and opportunities for the next decade," Frontiers in Oncology, vol. 10, p. 1727, 2020.

[37] G. Terrero and A. C. Lockhart, "Role of immunotherapy in advanced gastroesophageal cancer," Current Oncology Reports, vol. 22, no. 11, p. 112, 2020. 